\title{
Image Content Dependent Compression of Polarimetric SAR Data
}

\author{
Ursula BENZ, Jens FISCHER, Gunther JÄGER \\ Deutsches Zentrum für Luft- und Raumfahrt e.V. \\ Postfach 11 16, D-82230 Wessling, Germany \\ Tel: 49815328 2312, Fax: 49815328 1449; E-mail: Ursula.Benz@dlr.de
}

\begin{abstract}
This paper presents a novel technique for flexible SAR image compression in wavelet domain. Two approaches are considered: a) Compression leading to a scene independent, very homogenous signal-to-distortion noise ratio and constant phase error. Reconstruction quality varies - dependent from the required compression ratio - from good visible quality to nearly lossless reconstruction. b) Compression leading to high reconstruction quality for regions of interest and high compression ratio on background regions.
\end{abstract}

\section{INTRODUCTION}

Polarimetry and interferometry are most important remote sensing applications of SAR systems. They allow detailed extraction of information and the derivation of precise digital elevation models. A disadvantage is the huge data rate associated with those applications. Instead of one single data set, two, four or even eight channels of complex data have to be transmitted. Data compression becomes mandatory.

Performance of data compression can be measured according to two different strategies: 1) Application independent: No disadvantages for any application are given due to data compression. 2) Application dependent: No disadvantages for a certain application are given. Any drawbacks for other applications are not considered.

Usually the first strategy is followed, because interest of most data providers is to serve a large user community with different applications. This necessity is emphasized in the survey of on-board image compression for space applications [8], although the author mentions that future significant improvements of compression ratio are only achievable with selective compression.

Especially in IGARSS last year there appeared several approaches for selective compression with large benefits for the considered application. Compression adapted to transmission of data for sea ice navigation [6] leads to high compression ratio and good visual quality of the image. Another approach [1] considers separate compression of textured and nontextured regions with the assumption that either is of interest and the other is background. In the paper, textured regions are considered as background and replaced by their mean value while regions of interest are coded using compression in 1D wavelet domain. Other typical applications of selective com- pression are developed for military applications. Only the data of distinct man-made targets have to be carefully transmitted, while the background can be compressed significantely to ensure a narrow bandwidth for transmission.

All these developments are specific for an application and are therefore not suited for implementation in generic systems. Here we follow a different strategy to accommodate both: High compression ratio for specific applications and sufficient image quality for a large variety of applications. We assume that data storage itself won't be the limiting constraint in future systems due to rapid development of storage technology. Thus only a moderate compression ratio (up to 10) for storage is required. However, transmission bandwidth and transmission time will be tight goods. Additionally, with exploding data archives, dedicated search will be more and more time consuming.

Therefore we suggest to emphasize only selective decompression for adaptive transmission and reconstruction. Data compression itself is kept the same for all considered applications.

Thereto we compress the data using our flexible approach [2] to fulfill both, the requirements of the storage capacity and the considered applications. If no constraints are given, the data are compressed in a nearly lossless way. Thus even very high image qualities for complex interferometric and polarimetric computations are ensured. The algorithm is shortly described in chapter 1. Prior to data compression preclassification in basic object classes is performed. The classification map is stored in addition to the data and enables content based search in data archives. Classification can be performed in many ways. Important properties seems to us non-sensitivity to (speckle) noise and parameter variations. We propose a fuzzy classification described in chapter 2 . This classification map is used to control data transmission and decompression (chapter 3). It allows selection of the data set of interest and furthermore selection of foreground and background. Selection of regions of interest can be performed sequentially up to a full high-quality reconstruction of the data set. Thus transmission time and transmission bandwidth are minimized for the specific application. Flexibility for many applications is maintained due to the high-performance compression algorithm leading to less than $2^{\circ}$ phase error and more than $30 \mathrm{~dB}$ signal-to-noise ratio for full reconstruction $(\mathrm{cr}=10)$. 


\section{DATA COMPRESSION}

We apply a flexible SAR image compression algorithm. Depending on the image quality requirements, compression mode and compression ratio can be modified. Here, we consider the highly phase preserving mode of the algorithm. The complex data are compressed in cartesian format with a maximum compression ratio of 10 and a minimum compression ratio of 3 leading to a signal-to-distortion noise ratio (SDNR) of $30 \mathrm{~dB}$ and $65 \mathrm{~dB}$ (phase error less than $2^{\circ}$ degree and $0.1^{\circ}$ degree), respectively. Neither the correlations between neighbored pixels are changed nor geometric and radiometric resolution. No difference is given for cross and copolarized channels.

Thus, even sophisticated evaluations and feature extractions on those data sets for polarimetric and interferometric applications are possible without any degradations.

The algorithm uses wavelet transform to efficiently code the wavelet coefficients where in contrary to many implementation also the coarse scale image is lossy coded. This allows - for a given bit rate - to allocate more bits to the higher subbands. This is important to preserve the details and the phase information, because important information is also represented in the high frequency subbands if wavelet transform is applied on real and imaginary part of single-look complex SAR data.

The transform coefficients in each wavelet subband are quantized using a scalar block adaptive linear quantizer.

The wavelet subbands are divided into $\mathrm{N}$ quantization blocks $q_{i}, i: 1: N$. To ensure approximately constant statistic within one block $q_{i}$, the block size is small, e.g. 8 by 8 samples. The quantizer is adapted to the dynamic range $d_{i}$ of

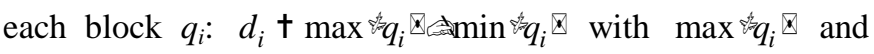
$\min \psi q_{i} \otimes$ maximum and minimum value within quantization block $q_{i}$, respectively. Dynamic range $d_{i}$ is divided into equally spaced intervals $I_{m}, I_{m}, m: 1: M_{i}$. The number $M_{i}$ is given by the available bit number $b_{i}$ for quantization of block $q_{i}: M_{i}+2^{b_{i}}$.

In standard mode all quantization blocks $q_{i}$ within one wavelet subband are quantized using the same bit number. Bit allocation is evaluated based on the ac energy distribution within the subbands and optimized in an iterative process to gain minimum quantization distortion. The average bit num-

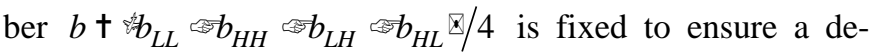
sired compression ratio.

Decompression requires transmission of header information: Bit mask $B$ and for each quantization block maximum

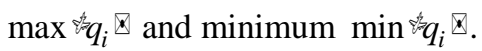

In adaptive mode the slight blocking effects around point targets and edges due to the quantization blocks are reduced by separate treatment of very high wavelet coefficients in the subbands [2].

\section{CLASSIFICATION MAP}

We derive a classification map for basic classes, e.g. urban, high vegetation (forest), medium and low vegetation and smooth surfaces using a supervised fuzzy learning system. In our simulation example we applied the fuzzy nearest prototype algorithm [7], which is implemented in our fuzzy interactive analysis system for SAR images FIAS [3]. To this end, we chose as feature vectors the ordered intensities of a 7 by 7 neighbourhood centered at the considered pixel. We selected 30 test samples for each of the five classes. After ordering the windows to 49-dimensional vectors $a_{i}^{\text {class }}+\left(a_{1}^{i}, \ldots, a_{49}^{i}\right)$ with $a_{1}^{i}: \ldots: a_{49}^{i}, i \dagger 1, \ldots, 30$, we averaged these vectors for each class to produce a typical test sample $T^{\text {class }}+\longrightarrow_{i+1}^{30} a_{i}^{\text {class }}$. The values of the components of $T^{\text {class }}$ are considered to be typical for the values of the class and will be used for quantization later on.

For classification the ordered feature vector $v \dagger\left(v_{1}, \ldots, v_{49}\right)$ of a pixel $p$ is compared to the typical test samples $T^{\text {class }}$ and a grade of membership is computed with the formula

$$
m(p, \text { class })=\frac{1}{d\left(v, T^{\text {class }}\right)} / \sum_{\text {class' }} \frac{1}{d\left(v, T^{\text {class }}\right)},
$$

which is interpreted as similarity of the pixel to the class. This is the fuzzy output for a pixel. Defuzzyfication with the rule

$p$ is of class $k$ if $m(p$, class $k) \geq m(p$, class $i)$ for all $i$ and $m(p$, class $k) \geq t$,

where $t$ is a classification threshold, leads to a crisp classification.

Up to now supervised classification is applied, however future studies will consider automatic refining of the search patterns to compensate varying data take conditions.

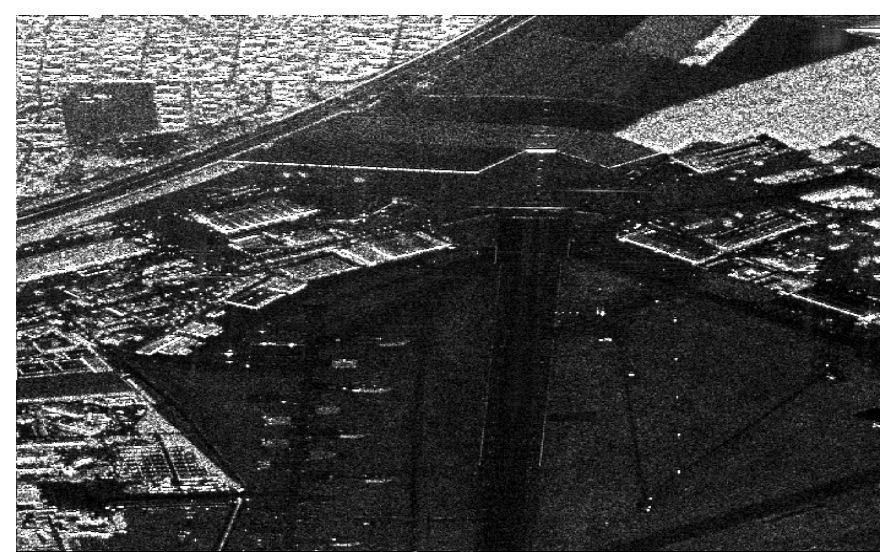


Fig.1: L-band polarimetric E-SAR data set, 64 bpp

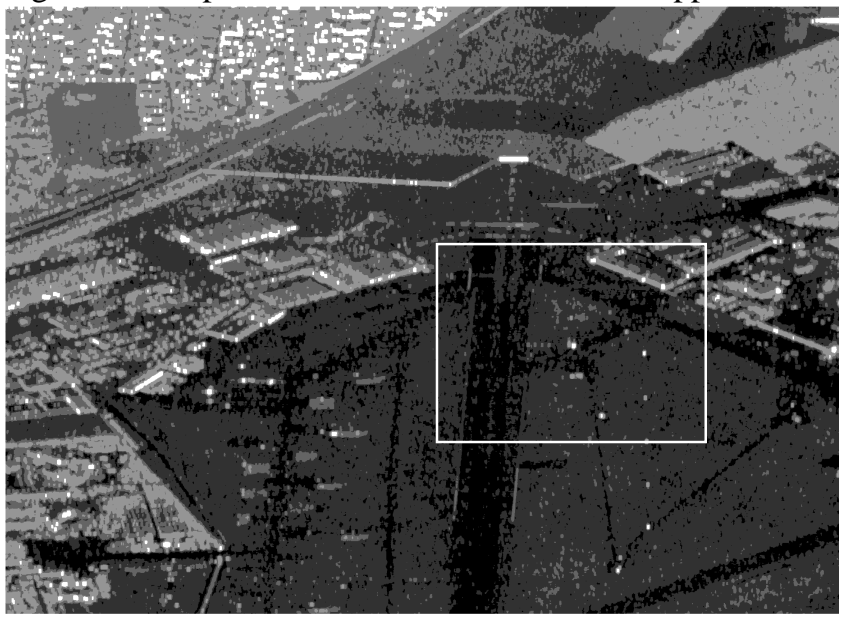

Fig. 2: Classification map, 3 bpp

In our example we achieve an overall classification accuracy of more than $80 \%$. (kappa: 0.78 , prod. acc. for smooth surface, veg.1, veg.2, forest, urban equals $0.9,0.65,0.99,0.91$ and 0.45 , fuzzy accuracies [4] give slightly worse values, but are here of minor interest.)

The classification map is an additional header for data compression with $3 \mathrm{bpp}$, but it is a valuable information on its own for data search and progessive data transmission. Fortunately, the classification map can easily be further compressed due to the high correlation between neighboured pixels. Common compression and decompression methods can be efficiently used.

\section{DATA TRANSMISSION AND DECOMPRESSION}

In the transmission chain, classification maps (fig. 2) are the first representations of the data sets in a data volume. The user can select on this bases which data set he is actually interested in. For some users the information delivered by this map can be already sufficient.

In most applications the user is only interested in some of the basic classes. Only those classes are reconstructed using the maximum number of bits.

The reconstructed values replace the classification map. The remaining background is only used to locate the regions of interest on the scene (fig. 3). On these regions the high image quality described is achieved.

Additional regions can be reconstructed sequentially with high quality according to the requirements of the user. If finally all classes are selected, the whole data set is transmitted without any measurable degradations on the object classes.

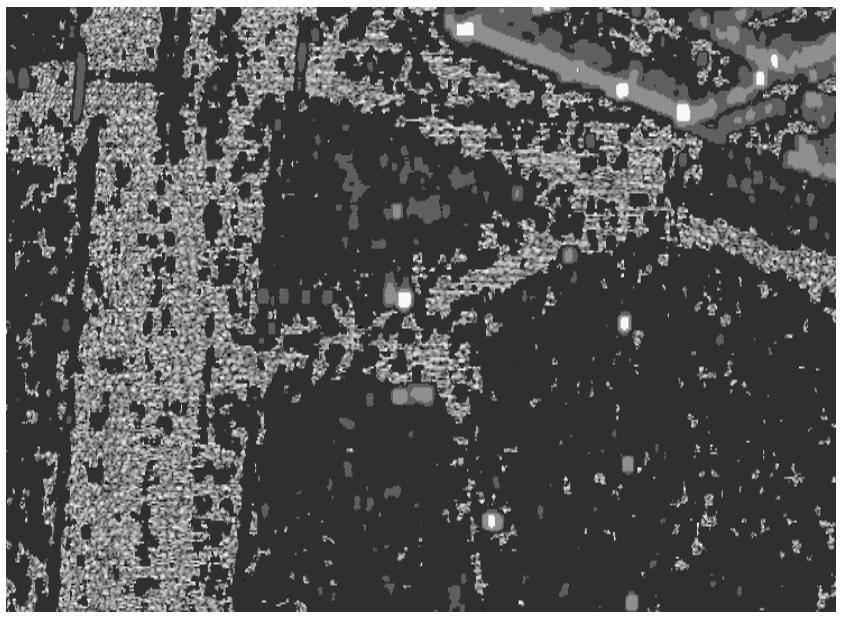

Fig. 3: Regions of interest (here, smooth surface) are reconstructed, enlargement of marked rectangle in fig. 2 .

\section{CONCLUSIONS}

This approach is very flexible and allows both, generic data compression and high compression ratio for data transmission.

In the presented example we don't take advantage from the classification map for data compression itself. However, similar to the approach for multispectral data by [Gelli] the segmentation in statistically more homogeneous regions can be used. Instead of using small quantization blocks with each of them requiring additional header information, larger blocks or even all the pixels of one class can be quantized using one adapted quantizer. The information about the chosen quantizer for a pixel is already given by the classification map. No additional header is necessary. Furthermore, classification considers the specific data statistics. The typical patterns found during training can be used for quantizer design.

\section{REFERENCES}

[1] Ancis, M., Murroni, M., Giusto, D., "Region-Based Temote-Sensing Image Compression in the Wavelet Domain", Proc. of IGARSS 99 vol. 4, pp. 2054-2057

[2] Benz, U., Fischer, J., "Adaptive SAR Image Compression in Wavelet Domain", Proc. of EUSAR 2000, to be printed

[3] Benz, U., Jäger, G.: "Multichannel Classification of SAR-Images Using Fuzzy Logic", Proc. $4^{\text {th }}$. Int. Airborne Remote Sensing Conference, Ottawa, Ontario, Canada, Vol. II, 857-866, 1999

[4] Jäger, G., Benz, U., "Measures of Classification Accuracy based on Fuzzy Similarity", TGARS, to appear

[5] Gelli, G., Poggi, G., Ragozini, A., Proc. of IGARSS 99, vol 2., pp. 1167-1171

[6] Karvonen, J. Similä, M., "Wavelet-Based Visual coding of Sea Ice SAR Images", Proc. of IGARSS 99, vol. 4, pp. 2254-2257

[7] Keller, J.M., Gray, M.R., Givens, J.A.: "A Fuzzy K-Nearest Neighbor Algorithm", IEEE Trans. Syst., Man, Cybern., vol. 15, no. 4, pp. 580-585, 1985

[8] Lambert-Nebout, C., "A survey of on-board image compression for CNES space missions", Proc. of IGARSS 99, vol. 4, pp. 2032 - 3035 